\title{
ICP curve morphology and intracranial flow-volume changes: a simultaneous ICP and cine phase contrast MRI study in humans
}

\author{
Mårten Unnerbäck ${ }^{1}$ (1) · Johnny T. Ottesen ${ }^{2} \cdot$ Peter Reinstrup $^{3}$ \\ Received: 1 September 2017 / Accepted: 12 December 2017 / Published online: 22 December 2017 \\ (C) The Author(s) 2017. This article is an open access publication
}

\begin{abstract}
Background The intracranial pressure (ICP) curve with its different peaks has been extensively studied, but the exact physiological mechanisms behind its morphology are still not fully understood. Both intracranial volume change $(\triangle \mathrm{ICV})$ and transmission of the arterial blood pressure have been proposed to shape the ICP curve. This study tested the hypothesis that the ICP curve correlates to intracranial volume changes.

Methods Cine phase contrast magnetic resonance imaging (MRI) examinations were performed in neuro-intensive care patients with simultaneous ICP monitoring. The MRI was set to examine cerebral arterial inflow and venous cerebral outflow as well as flow of cerebrospinal fluid over the foramen magnum. The difference in total flow into and out from the cranial cavity (Flow tot $_{\text {) }}$ over time provides the $\triangle \mathrm{ICV}$. The ICP curve was compared to the $\mathrm{Flow}_{\mathrm{tot}}$ and the $\triangle \mathrm{ICV}$. Correlations were calculated through linear and logarithmic regression. Student's $t$ test was used to test the null hypothesis between paired samples.

Results Excluding the initial ICP wave, P1, the mean $R^{2}$ for the correlation between the $\triangle \mathrm{ICV}$ and the ICP was 0.75 for the exponential expression, which had a higher correlation than the linear $(p=0.005)$. The first ICP peaks correlated to the initial peaks of Flow tot $_{\text {with }}$ a mean $R^{2}=0.88$.

Conclusion The first part, or the P1, of the ICP curve seems to be created by the first rapid net inflow seen in Flow tot while the rest of the ICP curve seem to correlate to the $\triangle \mathrm{ICV}$.
\end{abstract}

Keywords Intracranial pressure $\cdot$ Cerebral blood flow $\cdot$ Magnetic resonance imaging $\cdot$ Intracranial compliance

$\begin{array}{ll}\text { Abbreviations } \\ \text { aCBV } & \text { Arterial cerebral blood volume } \\ \text { CBFa } & \text { Arterial cerebral blood flow } \\ \text { CBFv } & \text { Venous cerebral blood flow } \\ \text { CBV } & \text { Cerebral blood volume } \\ \text { CSF } & \text { Cerebrospinal fluid } \\ \text { CSFF } & \text { Cerebrospinal fluid flow } \\ E & \text { Elastance coefficient } \\ \text { Flow }_{\text {tot }} & \text { Total flow into and out from the cranial cavity }\end{array}$

Mårten Unnerbäck

Marten.Unnerback@skane.se

1 Department of Clinical Sciences Lund, Intensive Care and Perioperative Medicine, Lund University, Skane University Hospital, Malmö, Sweden

2 Department of Science and Environment, Roskilde University, Roskilde, Denmark

3 Department of Clinical Sciences Lund, Department of Neurosurgery, Lund University, Skane University Hospital, Lund, Sweden

$\begin{array}{ll}\text { ICP } & \text { Intracranial pressure } \\ \mathrm{ICP}(\Delta \mathrm{ICV}) & \text { ICP as a function of } \Delta \mathrm{ICV} \\ \mathrm{ICV} & \text { Intracranial volume } \\ \mathrm{MRI} & \text { Magnet resonance imaging } \\ P_{1} & \text { pressure coefficient } \\ \mathrm{SD} & \text { Standard deviation } \\ \mathrm{TR} & \text { Repetition time } \\ \mathrm{vCBV} & \text { Venous cerebral blood volume } \\ \Delta \mathrm{ICV} & \text { Intracranial volume change } \\ \Delta \mathrm{ICV}_{\max } & \text { Maximal intracranial volume change }\end{array}$

\section{Introduction}

Lundberg [22] introduced the continuous monitoring of the intracranial pressure (ICP) in 1960 and this progress is today one of the most important aspects within neuro-intensive care [12]. The ICP curve follows the cardiac cycle and usually displays a variable amount of peaks. The peaks were initially interpreted as secondary to heart valves opening and closing during the 
cardiac cycle. Usually there are four peaks and Gega et al. proposed naming them $\mathrm{P} 1, \mathrm{P} 2, \mathrm{P} 3$, and so on $[11,16]$.

Early investigators pointed toward the possibility that the ICP curve is mainly shaped by the arterial blood pressure wave traveling through the arteries and then being transferred into the intracranial cavity $[9,15]$. To support this theory, it was early shown that extirpation of the plexus arachnoideus dampened the ICP curve, a finding that was interpreted as the result of less pressure transferring from the arteries to the intracerebral ventricles [9]. Other investigators concluded that it is the venous pressure that shapes the ICP curve [18]. Experiments in dogs lead to the conclusion that both the arterial and venous pressure waves affected the ICP curve [2].

Since the cranial cavity is a closed compartment, the ICP curve could be a product of intracranial volume (ICV) changes [3]. Previous investigations with phase contrast MRI found changes in ICV over the cardiac cycle, by measuring the arterial inflow; venous outflow, and cerebrospinal fluid (CSF) flow over the foramen magnum $[3,5,8]$. Initially, the arterial inflow supersedes the venous and CSF outflow generating an increase in ICV. However, the Monroe-Kellie doctrine states that the intracranial volume cannot change due to the incompressibility of the intracranial content, so if the measured change in ICV volume due to blood and CSF flow is true, there must be another change in intracranial volume to compensate for this. Through dynamic MRI technology, it has been shown that the intracranial parenchyma moves pulsatile in a rostral-caudal direction during the cardiac cycle [17]. Since the ICV changes are very small, it is feasible that this motion compensates the volume change, dampening ICP changes. This change in ICV may however not solely explain the morphology of the ICP curve since the change in ICV is slower in onset, peaks much later in the cardiac cycle than the ICP curve, and lacks the characteristic peaks [3].

It has been shown that the intracranial flow initially rises due to the arterial inflow and later on falls as the CSF and venous outflow compensates increasingly for this [3]. This generates a minimal but rapidly increasing ICV change $(\triangle \mathrm{ICV})$. This rapid flow could produce a substantial change in ICP since the only compensatory mechanism would be a slightly temporally delayed movement of the intracranial parenchyma caudally driven by the rise in ICP [17].

Although much work has been done regarding the intracranial compliance and compensatory mechanisms [20, 21, 27-29], less has been reported about the physiological mechanisms underlying the ICP changes over a cardiac cycle $[3,13$, $31,32]$.

We therefore wanted to investigate the flow in and out of the cranial cavity during a pulse cycle, the resulting $\triangle \mathrm{ICV}$, and correlate it to simultaneous measured intraventricular ICP recordings in neuro-intensive care patients.

\section{Materials and methods}

\section{Inclusion criteria}

Ethical approval for the study was granted by the Regional Ethical Review Board at Lund University. (2014/403) Patients admitted to the neuro-intensive care unit at the Skånes University Hospital during the period 2014-2017, monitored with an intra ventricular ICP catheter and clinical requirement of a MRI investigation were included. Patients with a non-intact cranial cavity, except for the insertion hole of the ventricular catheter, were excluded.

\section{MRI examinations}

MRI examinations were in all cases performed using a Philips Intera 1.5T. Slices, placed just under the foramen magnum perpendicular to the vessel, were $6 \mathrm{~mm}$ thick and a $256 \times$ 128 matrix was used. The velocity encoding value was set to $90 \mathrm{~cm} / \mathrm{s}$ for blood flow and $8 \mathrm{~cm} / \mathrm{s}$ for CSF during the phase contrast MRI examination. The flip angel was set to $15^{\circ}$. TR was $26 \mathrm{~ms}$. Each cardiac cycle was sampled at 30 to 35 time points and the total examination time was $2 \mathrm{~min}$. The arterial and venous blood flow was measured immediately before the CSF measurements, during a physiological stable time period.

All MRI examinations were analyzed using the freely available software SEGMENT v 2.0 R5432 [19]. One examiner, blinded to the ICP curves, performed the analysis of the MRI examinations. The region of interests of the internal carotid arteries, vertebral arteries, internal jugular veins, and the cerebrospinal canal were outlined manually and the flow were acquired in the phase contrast images pixel by pixel with a temporal resolution of 30 to 35 slices per cardiac cycle. The total flow in the carotid and vertebral arteries was summarized yielding the arterial cranial inflow. The flow in the internal jugular veins was summarized for estimation of the cranial venous outflow.

Venous outflow from the intracranial compartment may take different paths out of the cranial cavity and the proportion flowing through the internal jugular veins varies between individuals [14]. In order to determine the total venous outflow, the jugular venous outflow was multiplied by a factor to equal the arterial inflow over a cardiac cycle [3].

The flow during the cardiac cycle $\left(\right.$ Flow $\left._{\text {tot }}\right)$ was calculated at each time point by subtracting venous and CSF outflow from arterial inflow. The change of the intracranial volume over time was calculated by multiplying Flow tot $_{\text {with time, }}$ resulting in a volume change and then summarizing these volume changes over time, described in the equation:

$\Delta I C V(\tau)=\int_{\tau_{0}}^{\tau}(C B F a(t)-C B F \nu(t)-C S F F(t)) d t$

where $\triangle \mathrm{ICV}$ is the change in intracranial volume, $\mathrm{CBF}$ is the arterial cerebral blood flow, $\mathrm{CBFv}$ is the venous cerebral blood flow, and CSFF is the reverberating flow of CSF 
through the foramen magnum.

\section{ICP measurements}

The ICP had in all patients been measured with a 8-F tunneled intraventricular catheter (HanniKath, Smiths Medical Deutschland $\mathrm{GmbH}$ ), inserted through a burr hole cranially. The catheter had been connected to a CSF drainage set with a microtransducer (HanniSet, Smiths Medical Deutschland $\mathrm{GmbH})$. The transducer was zeroed against atmospheric pressure at the uppermost point of the cranium. The signal from the ICP pressure transducer was taken out of the MRinvestigation room through a radiofrequency filter at the penetration panel in the shielding enclosure into the MR-control room. In the control room, the values were digitally registered with a Philips Intellivue MP70 STAD at a sampling rate of $125 \mathrm{~Hz}$ and stored in a database. The ICP curves registered during the MRI examinations were identified and analyzed manually. All ICP curves during one respiratory cycle were summarized and a mean ICP curve calculated. This ICP curve was compared to the concurrent $\triangle \mathrm{ICV}$ derived from the MRI examinations using the lowest ICP and the lowest $\triangle \mathrm{ICV}$ as starting point.

\section{Statistical analysis}

All statistical analysis was performed using IBM SPSS Statistics for Windows, Version 22.0. (IBM Corp, USA) All values are given as mean and standard deviation. Linear regression was used to establish goodness of fit and to establish the optimal expression, either linear or logarithmic. Student's $t$ test was used to test the null hypothesis between paired samples.

All values are presented as Mean \pm SD unless stated otherwise.

\section{Results}

Mean age of the included 10 patients was $48.6 \pm 11.4$ years and 9 were male. The mean ICP value of the examinations was $16 \pm 10 \mathrm{mmHg}$. Maximum $\Delta \mathrm{ICV}\left(\Delta \mathrm{ICV}_{\max }\right)$ was $0.68 \pm$ $0.33 \mathrm{ml}$.

Total flow varied over time, with typically two or more peaks over a cardiac cycle. (Fig. 1).

Using linear regression, there was a correlation between ICP and $\triangle \mathrm{ICV}, R^{2}=0.41 \pm 0.14$. The correlation was strengthened using a logarithmic regression line, $R^{2}=0.47 \pm$ 0.13 . The correlation between ICP and $\triangle \mathrm{ICV}$ using logarithmic regression was stronger compared to using a linear regres$\operatorname{sion}(p=0.005)$.

The values representing $\mathrm{P} 1$ can be clearly identified as outliers when ICP is plotted against $\triangle \mathrm{ICV}$ (Fig. 2). In all
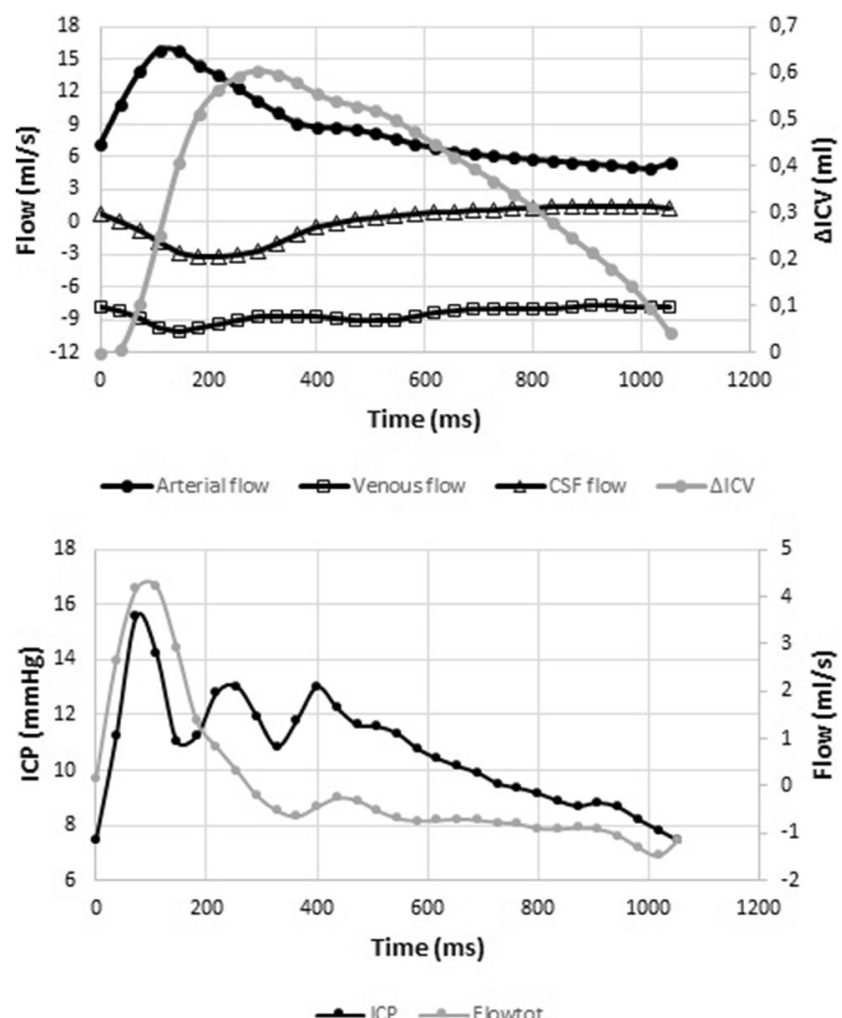

Fig. 1 The upper graph shows arterial flow, venous flow, CSF flow, and the resulting intracranial volume change $(\triangle \mathrm{ICV})$ over time. The lower graph shows the total flow $\left(\right.$ Flow $\left._{\text {tot }}\right)$ and the ICP curve. All data are from the same individual and plotted over the same timeline in the two graphs

examinations, but one, the correlation between $\triangle \mathrm{ICV}$ and ICP was reinforced when P1 was excluded. Using a logarithmic expression excluding P1 resulted in a mean $R^{2}$ of $0.75 \pm$ 0.15 , statistically significant compared to not excluding P1 $(p=0.001)$.

Plotting the $\triangle \mathrm{ICV}$ against ICP resulted in a graph where the $\triangle \mathrm{ICV}$ curve follows in close proximity of the ICP curve, except for the initial P1peaks (Fig. 3).

Comparing the total flow values during the initial peak of the flow curve with the ICP values of the P1 resulted in a

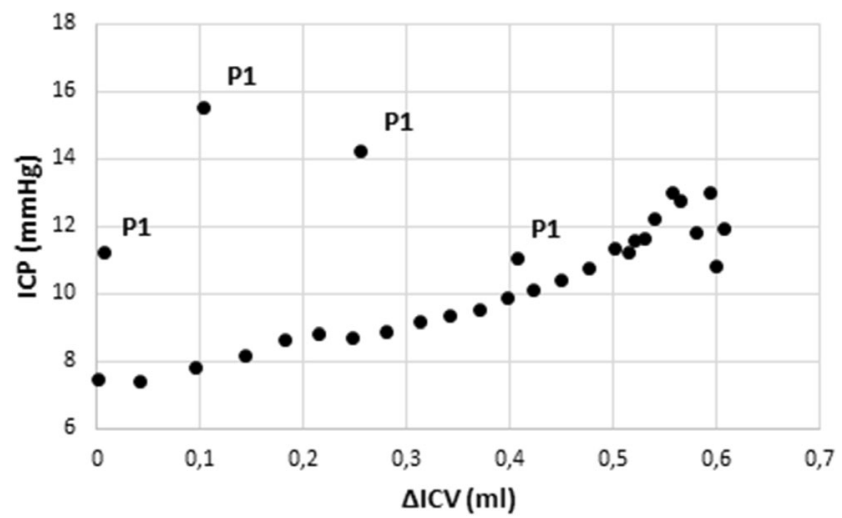

Fig. 2 Regression plot of intracranial pressure (ICP) against intracranial volume change $(\triangle \mathrm{ICV})$ in one individual. The points corresponding to the initial peak (P1) are apparent as outliers and marked "P1" 

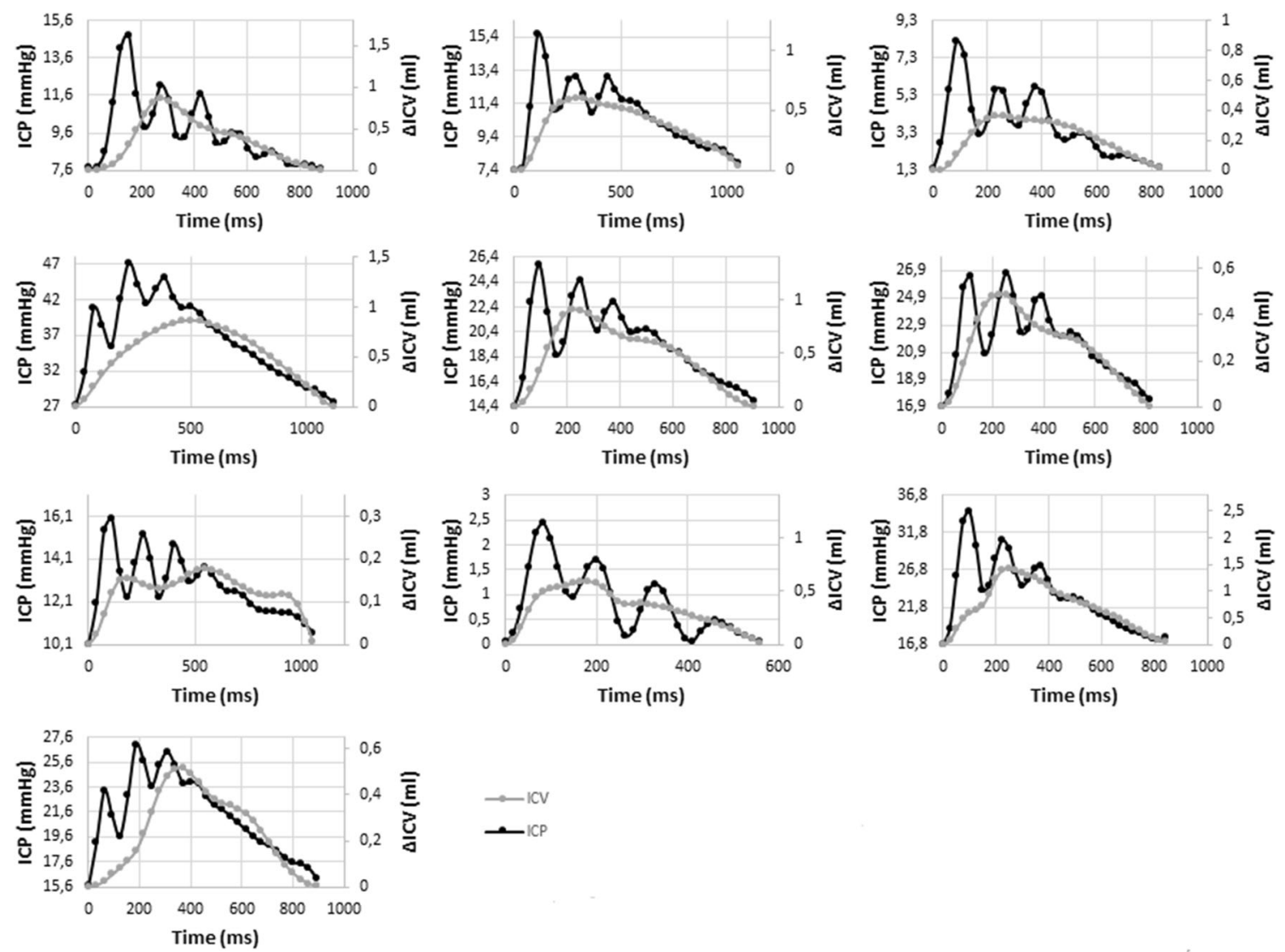

Fig. 3 Intracranial pressure (ICP) and $\triangle \mathrm{ICV}$ plotted against time for all examinations

correlation with a mean $R^{2}$ of $0.88( \pm 0.10)$. The mean temporal delay between the first flow peak and P1 was $22 \pm 28 \mathrm{~ms}$.

\section{Discussion}

The ICV includes three volumes that can change rapidly during a pulse stroke, the cerebral blood volume (CBV), which can be divided in arterial cerebral blood volume (aCBV) and venous cerebral blood volume (vCBV), as well as the cerebrospinal fluid (CSF). We hypothesized that the ICP curve morphology could be a result of the ICV change over time as well as an effect of the rapid inflow into the intracranial space. The data from this study shows a strong exponential correlation between the ICP changes and $\triangle \mathrm{ICV}$. To our knowledge, this link between concomitant ICV and ICP changes over the cardiac cycle has been proposed, but not fully demonstrated $[3,7,30]$. In a transcranial Doppler (TCD) study, Carrera et al. showed a relation between $\triangle \mathrm{CBV}$ and ICP [13] when assuming a constant venous outflow based on previous research [1]. However, several studies have demonstrated that the venous outflow from the cranial cavity is pulsatile $[3,4,25]$ and Carrera et al. do not compensate for CSF fluctuations, as there was no measurement of
CSF flow from the cranial cavity through the foramen magnum secondary to the change in CBV. Consequently, it could be argued that they demonstrated a relation between changes in aCBV and changes in ICP [30].

The ICP curve should more correctly correlate to the $\triangle \mathrm{ICV}$ than the $\triangle \mathrm{CBV}$. Since venous outflow from the cranial cavity increases dependent on the arterial inflow into the cranial cavity and the CSF outflow begins as the CBV increase $[8,26]$, the $\triangle \mathrm{ICV}$ curve peaks later as compared to the method using a constant venous outflow and not taking CSF flows into account. Carrera et al. concluded that the initial part of the ICP curve correlated to the arterial blood pressures (ABP) systolic peak and the subsequent part correlated to $\triangle \mathrm{CBV}$ [13] or more correctly, as earlier described, to the $\triangle \mathrm{aCBV}$. Our findings support a multifactorial origin of the ICP curve morphology as the $\triangle \mathrm{ICV}$ correlated to the ICP in the later part of the curve, but with no correlation to the initial ICP peak.

The exponential relation between ICP and ICV is well known from previous studies; it has been described by the following equation $[7,21,24,27]$ :

$\mathrm{ICP}=P_{1} \times e^{\mathrm{E} \times \mathrm{ICV}}$

where $P_{1}$ represents a pressure coefficient, $E$ represents the elastance coefficient, and ICV equals the intracranial volume. 
This relationship has previously been used for studying the relationship between ICV and ICP over the cardiac cycle in humans $[13,32]$. Our data supports the hypothesis that the exponential nature of this relationship is true over the small volume changes taking place during the cardiac cycle, since the strongest relationship was found between $\triangle \mathrm{ICV}$ and the ICP curve when described as an exponential function.

There is some evidence that $\mathrm{P} 2$ and $\mathrm{P} 3$ are caused by changes in $\mathrm{aCBV}$ and therefore dependent on the elastance of the craniospinal system [13]. Examining the graph plotting $\triangle \mathrm{ICV}$ against ICP (Fig. 3), it seems that patients in our study with a P1/ P2 amplitude ratio above 0.9 had a slower rise in $\triangle \mathrm{ICV}$, which consequently peaked later during the cardiac cycle. This slow change was attributed to high venous and CSF outflow rates and not to low arterial inflow. The two individuals with elevated ICP levels, but without an elevated P2, did not have this slow change in $\triangle \mathrm{ICV}$. Although our study is too small to find a statistically significant difference, we find this interesting.

It has been suggested that the initial sharp peak (P1) is a result of the transfer of the arterial blood pressure into the cranial cavity $[9,11,15]$. Since the ICP curve with its multiple peaks does not fully resemble the arterial pressure curve, there must be other factors affecting it. Rapid changes of intracranial flow could also produce a sharp peak. As the content of the cranial cavity is virtually incompressible, a small increase in ICV would lead to a large rise in ICP, an effect that could be dampened by other less well-examined factors referred to as brain elastance. If there is an inertia in this dampening system, the inflow could cause a small but rapid change in ICV and thereby ICP, since the compensatory mechanisms are put in effect with a slight delay. As the $\triangle \mathrm{ICV}$ rate of change, i.e., Flow $_{\text {tot }}$, decelerates due to the venous and CSF outflow, the dampening become sufficient and the ICP falls rapidly resulting in a peak. The delay in the response to the raise in ICP could cause resonance in the system, which as a result could lead to the oscillations observed around the increased ICP due to the $\triangle \mathrm{ICV}$ increase. The subsequent peaks observed could thereby be the result of the interaction between arterial inflow, venous outflow, CSF flow over the foramen magnum, and the intracranial elastance. These changes are probably minor, which would explain why we couldn't measure them fully with the present method.

It is possible that a stronger correlation could have been found if we had chosen a regression line other than logarithmic or linear. This was considered, but we concluded that the logarithmic or linear lines served the purpose of this study best.

In this study, we did not have the possibility to record the ABP curve simultaneously. Since the ICP curve seems to be a compound of several different factors, it would be of interest to include this in future studies.

The technique for measuring the arterial, venous, and CSF flows, using MRI, has been used previously, but not in conjunction with simultaneous ICP recording. The MRI flow measurements have been demonstrated to have methodological errors of max $10 \%$. [10, 23] It should however be stressed that the volumes measured are small and minor errors could affect the data, probably in a systematic way. The changes in $\triangle \mathrm{ICV}$ measured are within what have been predicted to be in the physiological range [6]. Our conclusion is that the shape of the measured flow curve could be regarded as consistent with the shape of the actual flow curve.

The study has a limited sample size and further studies with this methodology could provide more robust and precise answers.

\section{Conclusion}

Our data suggests that the change in the ICP curve during a pulse stroke is composed from different cerebrovascular flow parameters. The first peak of the ICP curve correlated well to the initial arterial blood flow into the cranial cavity, opening for the possibility that the primary part of the ICP curve is, in some part, created by this flow. The latter parts of the ICP curve correlated to the $\mathrm{CBV}$ changes over the cardiac cycle, a correlation that seems to be of an exponential nature.

Funding This study received financial support from the Department of Intensive \& Perioperative Care, Skanes University Hospital and the Swedish National Training and Research Fund, Region Skåne, grant no 48451 .

\section{Compliance with ethical standards}

Conflict of interest None.

Ethical approval All procedures performed in studies involving human participants were in accordance with the ethical standards of the Lund University research committee (2014/403) and with the 1964 Helsinki declaration and its later amendments or comparable ethical standards. For this type of study, formal consent is not required.

Open Access This article is distributed under the terms of the Creative Commons Attribution 4.0 International License (http:// creativecommons.org/licenses/by/4.0/), which permits unrestricted use, distribution, and reproduction in any medium, provided you give appropriate credit to the original author(s) and the source, provide a link to the Creative Commons license, and indicate if changes were made.

\section{References}

1. Aaslid R, Newell D, Stooss R, Sorteberg W, Lindegaard KF (1991) Assessment of cerebral autoregulation dynamics from simultaneous arterial and venous transcranial Doppler recordings in human. Stroke 22:1148-1154

2. Adolph R, Fukusumi H, Fowler N (1967) Origin of cerebrospinal fluid pulsations. Am J Phys 212:840-846 
3. Alperin N, Lee S, Loth F, Raksin P, Lichtor T (2000) MRintracranial pressure (ICP) a method to measure intracranial elastance and pressure noninvasively by means of MR imaging: baboon and human study. Radiology 217:877-885

4. Alperin N, Lee S, Sivaramakrishnan A, Hushek S (2005) Quantifying the effect of posture on intracranial physiology in humans by MRI flow studies. J Magn Reson Imaging 22:591-596

5. Alperin N, Sivaramakrishnan A, Lichtor T (2005) Magnetic resonance imaging-based measurements of cerebrospinal fluid and blood flow as indicators of intracranial compliance in patients with Chiari malformation. J Neurosurg 103:46-52

6. Avezaat C, van Eijndhoven J (1986) Clinical observations on the relationship between cerebrospinal fluid pulse pressure and intracranial pressure. Acta Neurochir 79:13-29

7. Avezaat C, van Eijndhoven J, Wyper D (1979) Cerebrospinal fluid pulse pressure and intracranial volume-pressure relationships. J Neurol Neurosurg Psychiatry 42:687-700

8. Balédent $\mathrm{O}$, Henry-Feugeas M, Idy-Peretti I (2001) Cerebrospinal fluid dynamics and relation with blood flow: a magnetic resonance study with semiautomated cerebrospinal fluid segmentation. Investig Radiol 36:368-377

9. Bering E (1955) Choroid plexus and arterial pulsation of cerebrospinal fluid. Demonstration of the choroid plexuses as a cerebrospinal fluid pump. Arch Neurol Psychiatr 73:165-172

10. Bryant DJ, Payne JA, Firmin DN, Longmore DB (1984) Measurement of flow with NMR imaging using a gradient pulse and phase difference technique. J Comput Assist Tomogr 8:588593

11. Cardoso E, Rowan J, Galbraith S (1983) Analysis of the cerebrospinal fluid pulse wave in intracranial pressure. J Neurosurg 59: $817-821$

12. Carney N, Totten AM, O'Reilly C, Ullman J, Hawryluk G, Bell M et al (2017) Guidelines for the management of severe traumatic brain injury, fourth edition. Neurosurgery $80: 6-15$

13. Carrera E, Kim D, Castellani G, Zweifel C, Czosnyka Z, Kasprowicz M, Smielewski P, Pickard J, Czosnyka M (2010) What shapes pulse amplitude of intracranial pressure? J Neurotrauma 27:317-324

14. Doepp F, Schreiber SJ, von Münster T, Rademacher J, Klingebiel R, Valdueza J (2004) How does the blood leave the brain? A systematic ultrasound analysis of cerebral venous drainage patterns. Neuroradiology 46:565-570

15. Dunbar H, Guthrie T, Karpell B (1966) A study of the cerebrospinal fluid pulse wave. Arch Neurol 14:624-630

16. Gega A, Utsumi S, Iida Y, Iida N, Tsuncda S (1980) Analysis of the wave pattern of CSF pulse wave. In: Shulman K, Marmarou A, Miller J, Becker D, Hochwald G, Brock M (eds) Intracranial pressure IV. Springer, Berlin, pp 180-190

17. Greitz D, Wirenstam R, Franck A, Nordell B, Thomsen C, Ståhlberg F (1992) Pulsatile brain movement and associated hydrodynamics studied by magnetic resonance phase imaging. The Monro-Kellie doctrine revisited. Neuroradiology 34:370-380

18. Hamit H, Beall A, DeBakey M (1965) Hemodynamic influences upon brain and cerebrospinal fluid pulsations and pressures. $\mathrm{J}$ Trauma 5:174-184

19. Heiberg E, Sjögren J, Ugander M, Carlsson M, Engblom H, Arheden HD (2010) Design and validation of segment - a freely available software for cardiovascular image analysis. BMC Med Imaging 10:1
20. Lim S, Potts D, Deonarine V, Deck M (1973) Ventricular compliance in dogs with and without aqueductal obstruction. J Neurosurg 39:463-473

21. Löfgren J, Zwetnow N (1973) Cranial and spinal components of the cerebrospinal fluid pressure-volume curve. Acta Neurol Scand 49: $575-585$

22. Lundberg N (1960) Continuous recording and control of ventricular fluid pressure in neurosurgical practice. Acta Psychiatr Scand Suppl 36:1-193

23. Marks MP, Pelc NJ, Ross MR, Enzmann DR (1992) Determination of cerebral blood flow with a phase-contrast cine MR imaging technique: evaluation of normal subjects and patients with arteriovenous malformations. Radiology 182:467-476

24. Marmarou A, Shulman K, LaMorgese J (1975) Compartmental analysis of compliance and outflow resistance of the cerebrospinal fluid system. J Neurosurg 43:523-534

25. Müller L, Toro E (2014) Enhanced global mathematical model for studying cerebral venous blood flow. J Biomech 47:3361-3372

26. Raksin P, Alperin N, Sivaramakrishnan A, Surapaneni S, Lichtor T (2003) Noninvasive intracranial compliance and pressure based on dynamic magnetic resonance imaging of blood flow and cerebrospinal fluid flow: review of principles, implementation, and other noninvasive approaches. Neurosurg Focus 14(4):e4

27. Ryder H, Espey F, Kimbell F, Penka E, Rosenauer A, Podolsky B, Evans J (1953) The mechanism of the change in cerebrospinal fluid pressure following an induced change in the volume of the fluid space. J Lab Clin Med 41:428-435

28. Sklar F, Elashvili I (1977) The pressure-volume function of brain elasticity. Physiological considerations and clinical applications. J Neurosurg 47:670-679

29. Szewczykowski J, Sliwka S, Kunicki A, Dytko P, Korsak-Sliwka J (1977) A fast method of estimating the elastance of the intracranial system. J Neurosurg 47:19-26

30. Tain R, Alperin N (2013) Intracranial pressure dynamics are not linked to aqueductal cerebrospinal fluid stroke volume. J Appl Physiol 114:1645

31. van Eijndhoven J, Avezaat C (1986) Cerebrospinal fluid pulse pressure and the pulsatile variation in cerebral blood volume, an experimental study in dogs. Neurosurgery 19:507-522

32. Wåhlin A, Ambarki K, Birgander R, Alperin N, Malm J, Eklund A (2010) Assessment of craniospinal pressure-volume indices. AJNR Am J Neuroradiol 31:1645-1650

\section{Comments}

The field of pulse wave analysis has great potential in diagnosing and predicting ICP elevations in patients with TBI and other neurosurgical emergencies. But it needs basic studies of physiology like this to expand our understanding of the pulse wave propagation inside the cranium. Before we can compute models for predicting an ICP crisis, we need to understand why the intracranial pulse wave appears as it does in different conditions. This study correlates the volume change inside the cranium and correlates it to the measured ICP, which is an original idea, that moves our understanding forward.

Alexander Lilja-Cyron

Denmark 